\title{
Ultrasonic Fouling Detector Powered by Machine Learning
}

\section{Sillanpää, Tom Oskar Nikolai}

IEEE

2019

Sillanpää , T O N , Rauhala , T , Mäkinen, J M K, Rajani , C , Longi , K E , Klami , A , Salmi , A \& Haeggström , E 2019 , Ultrasonic Fouling Detector Powered by Machine Learning . in 2019 IEEE International Ultrasonics Symposium (IUS). IEEE International Ultrasonics Symposium , IEEE , pp. 1639-1642, IEEE International Ultrasonics Symposium , Glasgow , United Kingdom , 06/10/2019 . https://doi.org/10.1109/ULTSYM.2019.8925773

http://hdl.handle.net/10138/321739

https://doi.org/10.1109/ULTSYM.2019.8925773

acceptedVersion

Downloaded from Helda, University of Helsinki institutional repository.

This is an electronic reprint of the original article.

This reprint may differ from the original in pagination and typographic detail.

Please cite the original version. 


\section{Ultrasonic Fouling Detector Powered by Machine Learning}

\author{
Tom Sillanpää \\ Department of Physics, \\ University of Helsinki \\ Helsinki, Finland \\ tom.sillanpaa@helsinki.fi \\ Krista Longi \\ Department of Computer \\ Science, University of Helsinki \\ Helsinki, Finland
}

\author{
Timo Rauhala \\ Altum Technologies
}

Helsinki, Finland

Arto Klami

Department of Computer

Science, University of Helsinki

Helsinki, Finland

\author{
Joni Mäkinen \\ Department of Physics, \\ University of Helsinki \\ Helsinki, Finland
}

Ari Salmi

Department of Physics,

University of Helsinki

Helsinki, Finland

\author{
Chang Rajani \\ Department of Computer \\ Science, University of Helsinki \\ Helsinki, Finland
}

Edward Hæggström

Department of Physics,

University of Helsinki

Helsinki, Finland

\begin{abstract}
Guided waves can be used to monitor structural health in industrial pipelines, and e.g. allow detection of accumulated precipitation on the surface of pipe. Propagation of guided waves in a tubular structure carrying possible fouling can be separated from a clean structure due to variation in wave propagation properties at the fouled area. In addition, multiple propagation paths around the tubular structure allow locating the fouled areas. In this study, we obtained dispersion curves of a tubular structure loaded with a local fouling layer of different thickness by using numerical simulations. We combined the dispersion curve information with simulated and measured timesof-arrival of guided wave propagation to second order helicoidal paths and used a Gaussian Process machine learning approach to estimate location of fouling on a steel pipe.
\end{abstract}

Keywords-Guided wave, Fouling detection, $\mathrm{NDE}$

\section{INTRODUCTION}

Usual applications of guided waves (GW) for nondestructive evaluation (NDE) include inspection of corrosion [1], detection of flaws [2] and accumulation of dirt on the surfaces of, e.g. pipes [3] and rotating wind turbine blades [4]. The GW technique has been proposed to estimate fouling rates [3]. In industry, accumulation of fouling in structures (e.g. heat exchangers and pipelines) tends to decrease their efficiency and increase maintenance cost (possible process breaks due to cleaning). The GW-technique allows structural health monitoring (SHM).

In comparison with traditional ultrasound methods (pointby-point measurements), GW inspection provides a large detectable area with a single measurement, and due to low attenuation, they propagate long distances even in embedded structures such as buried or coated pipes. These properties permit GW inspection of pipelines where coating needs to be removed only at sensor locations. On the other hand, GW inspection in complex structures is challenging, and especially in topologies curving on to themselves. It is difficult to interpret signals caused by many possible propagating modes and paths in the certain structure. Especially for tubular structures, multipath propagation has been studied [5]-[8]. The multipath propagation due to tubular geometry allows the guided waves to propagate in multi-order helicoidal paths. These helicoidal waves may contain information about the spatial integrity or cleanliness of the pipe due to the distinguishable propagation paths.

Another property of GWs is their dispersive nature. Commonly, a dispersion curve displays the frequency dependence of phase speed or group speed (propagation speed of a certain mode). Depending on the boundary conditions, e.g. whether a structure is planar or tubular, the dispersive properties differ [9]. Also, if the surface of the structure is loaded with a fluid, coatings or an accumulation of precipitation, it alters the boundary conditions [10]. In these cases the GWs can become leaky, i.e. the propagating waves leak acoustic energy into the embedded media [10], which changes the dispersive properties of the system. For a tubular structure where an accumulation of dirt envelops the inner or outer surface of the tube, the group speed of some modes change, since the GWs leak also into the fouling layer. Consequently, the group speed of different modes may be different in a clean and in a fouled case. Analytical calculation of dispersion curves is hard for tubular structures that are fluid-filled and that carry local patches of fouling of particular thickness. The Finite element method (FEM) can be used to estimate the dispersion curves of a tubular structure [11]. In a FEM simulation, it is possible to add different material layers with arbitrary height into the pipe.

We combined a selective reception of the helicoidal waves with a machine learning approach. The method uses numerical dispersion curves obtained with the FEM based simulations and a Gaussian Process (GP)-model [12] to estimate fouled areas and rates. The fouling is modeled as smoothly varying layer of unknown thickness on the pipe surface. The posterior distribution of fouling is estimated based on the difference between experimental times-of-arrival (TOAs) of the circumferential wave packets and theoretical TOAs computed by FEM for a clean structure. As the difference is caused by the change in propagation velocity induced by the fouling, we use integral observations of the cumulative change along the propagation paths for posterior inference [12]-[14], to have a 
spatial map that indicates the rate of fouling at all locations on the surface, together with uncertainty estimates.

Based on these, we explored a way to develop a GW-based fouling detector for metal pipe inspection augmented with Artificial Intelligence (AI).

\section{MATERIALS \& METHODS}

\section{A. Experimental setup}

A commercial transducer (Karl Deutsch S24HB0.3$1.3 \mathrm{MHz}$ ) attached to a wedge was used to generate GWs into a steel pipe $(\mathrm{L}=2500 \mathrm{~mm}, \emptyset=155 \mathrm{~mm}$, thickness $=2 \mathrm{~mm})$ with an angle-of-incidence method $\left(\theta=55^{\circ}\right)$. Three-cycle Hanningwindowed signals were generated using an arbitrary waveform generator (Digilent Analog Discovery 2) and were amplified with a 500W amplifier (Amplifier Research 500A100A). The signals were received with an identical transducer using preamplifier (Panametrics 5660C) with a gain of $60 \mathrm{~dB}$ and captured with an oscilloscope (Lecroy wavesurfer 3014z) at 10 $\mathrm{MHz}$ sampling rate, averaging over 256 signals.

Artificial fouling was prepared by mixing water to a calcium hydroxide powder $(4: 5 \mathrm{v} / \mathrm{v})$ that was then manually added to the inner surface of the steel pipe in two different settings. Two kinds of experiments were conducted: (\#1), fouling was added at one location to the inner surface of the pipe, mimicking a clump or accumulation inside a pipeline (ellipsoid in shape with dimensions of $\mathrm{L}=28 \mathrm{~cm}, \mathrm{~W}=10 \mathrm{~cm}$ and central height $=1 \mathrm{~cm}$ ). The measurement was done as previously described, and the receiving transducer $(\mathrm{RX})$ was placed at 25 radial positions $(\mathrm{Z}=$ $70.3 \mathrm{~cm}$ and $\theta=[0-350]$ degrees around the pipe that does not contain water inside (Fig. 1).

Experiment (\#2) consisted of measurements, in which, GW signals were captured pre, during and post ultrasound two cleaning cycles. The cleaning equipment was provided by Altum technologies ${ }^{\circledR}$ consisting of a two-channel cleaning system attached at locations: in first cleaning cycle at $\mathrm{Z}=28$ and $56 \mathrm{~cm}\left(\theta=0^{\circ}\right)$ and $Z=14$ and $69 \mathrm{~cm}\left(\theta=0^{\circ}\right)$ in the second. $\mathrm{GW}$ generation (TX) was also done with a wedge and a $\mathrm{RX}$ transducer was positioned in parallel line $\left(\theta=0^{\circ}\right)$ with the wedge at distance $(\mathrm{D}=83.3 \mathrm{~cm})$ and a five-cycle Hanning-windowed signals were generated at $\mathrm{f}=500 \mathrm{kHz}$. Cleaning and

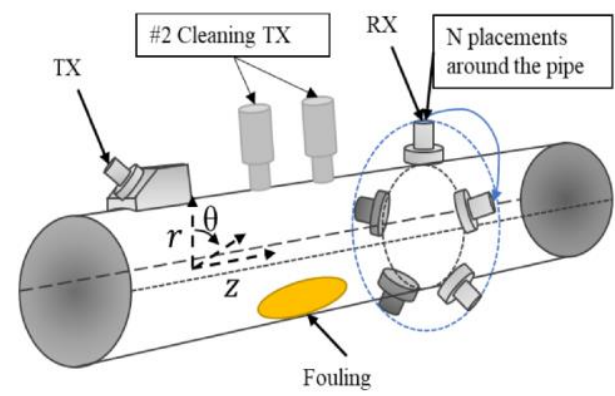

Fig. 1. Experimental setup used in the first measurement (1.). Ultrasonic pulses were sent with a wedge-transducer and recorded at a distance $(\mathrm{L}=$ $70.3 \mathrm{~cm}$ from front wall of wedge) from 25 positions $(2 \mathrm{~cm}$ radially apart from each other) around the pipe. An elliptical $(\mathrm{L}=28 \mathrm{~cm}, \mathrm{~W}=10 \mathrm{~cm}$, center at angle $\theta=160^{\circ}$ ) accumulation of fouling (Calcium hydroxide) was inserted to the inner surface of the pipe. The thickness profile was roughly parabolic in shape with a central height of $1 \mathrm{~cm}$. Two cleaning transducers that are used in the second measurement (2.) are also shown.

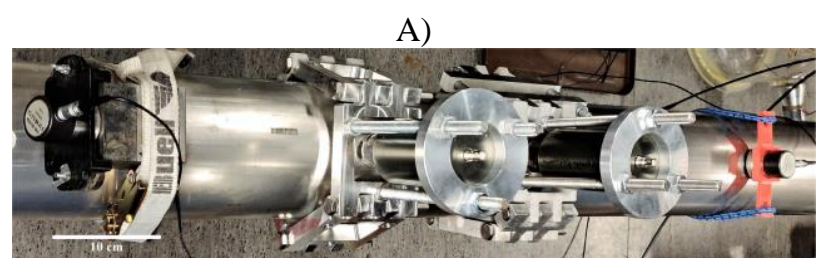

B)

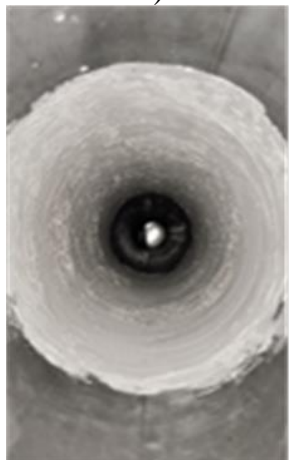

C)

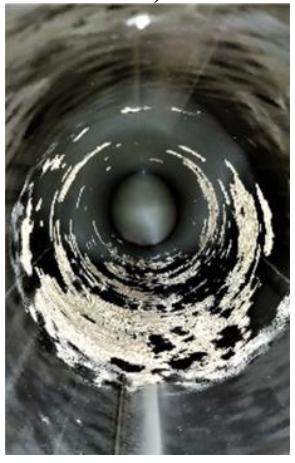

Fig. 2. A) Image of the setup in the second measurement. It contains a wedge at TX, two cleaning transducers attached to the pipe and an RX transducer placed in-line with the wedge $(\mathrm{D}=83.3 \mathrm{~cm}$ from wedge). $\mathrm{B})$ Fouling layer at inner surface of the pipe $(\mathrm{L}=70 \mathrm{~cm}$, thickness $=1.5$ $\mathrm{mm}$ ). C) Image post second cleaning cycle shows fully cleaned areas and residual fouling, which was not fully removed with these cleaning sets.

measurements were conducted with a water-filled pipe. The setup is displayed in (Fig. 2A), fouling layer (pre-cleaning) (Fig. 2B) and fouling remaining post second cleaning cycle (Fig. 2C).

In the first measurement GW signals were analyzed with MATLAB $®(R 2018 \mathrm{a})$ and the TOFs of a quasi- $\mathrm{S}_{0}$ mode were extracted by finding local maximum of smoothed envelopes of the squared signals with a time-window $\left(t_{\text {window }}= \pm 9 \mu \mathrm{s}\right)$ around the calculated theoretical arrival time of different helicoidal wave packets. These values were given as input to the machine learning-algorithm, as is explained in section II.C.

\section{B. FEM Simulations}

Finite element method simulations were used to determine dispersion curves for both the clean and fouled steel pipes. The simulations were done with COMSOL Multiphysics® (5.4). Dispersion curves were obtained using a mode analysis solver that calculates the possible eigenmodes of a 2D-cross-sectional geometry. This solver can be used to find out-of-plane

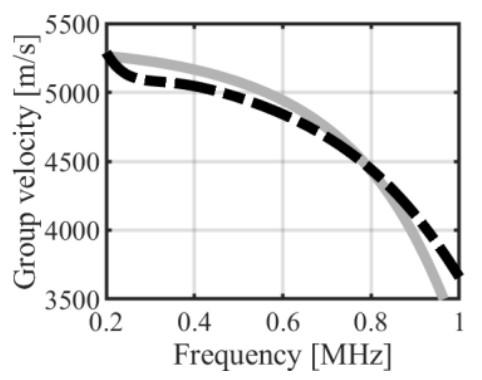

Fig. 3. Dispersion curves obtained with FEM from a pipe (gray line, $\varnothing=$ $155 \mathrm{~mm}$ and thickness $=2 \mathrm{~mm}$ ) without and with a fouling layer that envelops the inner surface of the pipe (black dashed line, fouling material $\mathrm{Ca}(\mathrm{OH}) 2$, fouling layer thickness $=7 \mathrm{~mm}$ was added on the inner diameter of the pipe). 
wavenumbers of an infinite pipe with an arbitrary cross-section at a certain given frequency [11]. In this case the frequency was swept throughout the range according to the experiments. Only a thin slice of the $2 \mathrm{D}$ cross-section of the pipe was modelled in order to reduce the geometrical modes that the solver finds. This allowed us to efficiently simulate the lowest order quasi-lamb wave modes. The material parameters were chosen from the COMSOL material library as steel for the pipe and elastic modulus $E=48 \mathrm{GPa}$ for Calcium hydroxide (fouling) was provided by [15] and Poisson's ratio $v=0.08$ by [16]. Material parameter sweep were conducted. This exercise indicated in that the variation in $E$ and $v$ from the choice of fouling material was insignificant.

\section{Artificial Intelligence}

We assumed that the amount of fouling on the surface is a smoothly varying unknown function that follows a Gaussian process $(\mathrm{GP})$ prior $\mathrm{f} \sim \mathrm{GP}\left(\mathrm{m}(\mathrm{x}), \mathrm{K}\left(\mathrm{x}, \mathrm{x}^{\prime}\right)\right)$, such that a collection of evaluations of the function at a finite set of points follows a multivariate normal distribution with mean function $\mathrm{m}(\mathrm{x})=0$ and covariance controlled by a kernel function $\mathrm{K}\left(\mathrm{x}, \mathrm{x}^{6}\right)$ [12]. We use the Matern 3/2 kernel [12] with length-scale $l$, output variance $o^{2}$ and noise variance $s^{2}$ controlling the smoothness and noise assumptions, and additionally assume that there is no fouling within $4 \mathrm{~cm}$ from the transducers. The posterior distribution of the function conditional on observations of linear operators of the function is available in analytic form [12]-[14].

We can only observe the function indirectly. Local fouling changes the propagation speed of the lamb-type helicoidal wave and at the transducer we observe the arrival time of the wave (Sec. II.A), influenced by the fouling along the whole trajectory. The difference between the observed arrival time and the theoretical arrival time for clean pipe (Sec. II.B) hence corresponds to a path integral of the group velocity change inducted by the fouling. By choosing a certain fd-product with a suitable transducer, we can in principle observe the arrival times for all modes for a few high-order helicoidal paths (propagation paths that circumnavigates the tubular structure $n>1$ times). The estimation of TOA differences is most reliable for the modes that arrive first, and hence we use the $S_{0}$ lamb-type mode for paths up to order $n=2$. The result of the AI module is full spatial map of fouling represented as distribution over fouling thickness at each location.

\section{RESULTS}

This section is divided into two parts: The first part displays the captured signals from two measurement settings, as described in II.A. The second part focuses on the results of estimated fouled area and rate provided with artificial intelligence, as mentioned in II.C.

\section{A. Experiments}

Lowest order modes of GW generated $(\mathrm{f}=300 \mathrm{kHz}$ ) into a steel pipe at one location propagated along several circumferential paths throughout a parallel axial distance of 70.3 $\mathrm{cm}$ (from wedge front to the center of receiving transducer). The receiving transducer $(\mathrm{RX})$ was placed at 25 radial positions (varying only the $\theta$ angle) around the pipe and the captured signals from a clean and from a fouled pipe are shown in (Fig.
4). From these signals the TOAs of helicoidal waves were determined and used as input to the machine learning part.

In the second measurement only the difference pre- and post ultrasonic cleanings were targeted without localization attempts. The signals captured during the experiment is shown in (Fig. 5). The line at the bottom shows a low amplitude signal from the fouled pipe (Fig. 2B). The wave packets of the signal correspond to $S_{0}$ lamb-type guided waves. Attaching cleaning transducers around the pipe (Fig. 2A) decreased the amplitude of these wave packets (second from bottom). After the first cleaning procedure $(\mathrm{t}=5 \mathrm{~min})$, an increase in the amplitude of the wave packets was noticed (third from bottom).

To ensure the cleaning result in the pipe, water was emptied prior to second cleaning sonication. The second sonication was conducted at the secondary placements of the cleaning transducers and the GW signals were recaptured. This again showed an increase in amplitude (blue line) and resembles closely the signal obtained from the clean pipe.

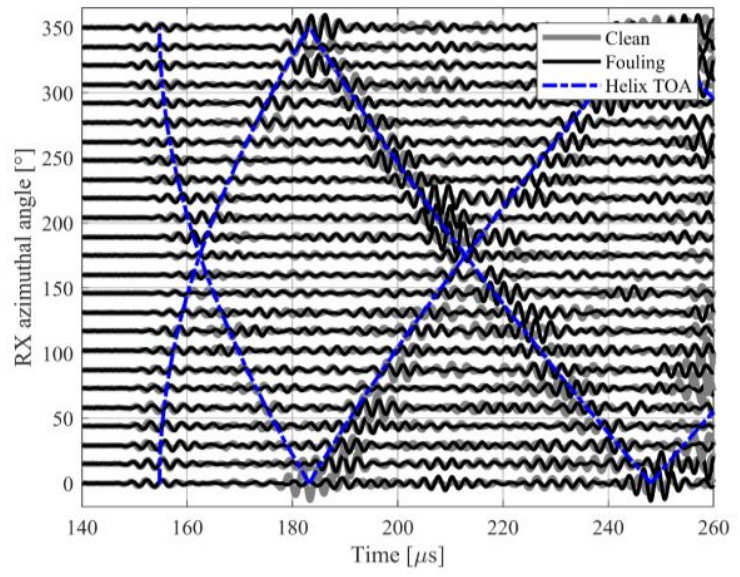

Fig. 4. Signals generated with one transducer attached to a wedge and guided waves propagated in a metal pipe a distance of $\mathrm{d}=70.3 \mathrm{~cm}$, where it was recorded around the pipe from 25 locations on $\theta_{\text {step }}=15^{\circ}$ with respect to each other at an equidistance plane from the wedge. Signals on top of each other from the clean (gray) and with an accumulation of fouling at pre-described location (black). The calculated TOAs of the circumferential wave packets (clockwise and counter-clockwise) are indicated with blue lines.

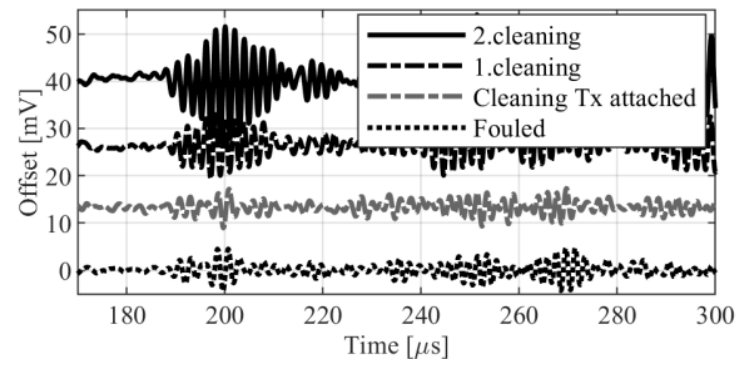

Fig. 5. Signals obtained in ultrasound cleaning procedure with the fouled pipe described in II.A ( $\mathrm{f}=500 \mathrm{kHz}, 5$-cycles). The amplitude of the first arrival wave packets of the signal (at bottom) was low compared to the amplitude of signals recorded after the first (third from bottom) and second (top) cleaning procedure. 


\section{B. Artificial Intelligence}

We first verify the AI model in silico, based on TOA differences obtained as numerical integrals of assumed known fouling, an ellipsoid of constant thickness. The result, depicted as probability of fouling exceeding half of the assumed thickness in Fig. 6, verifies that the used sensor array and the GP model can accurately localize the fouling in a low-noise condition. Fig. 7 presents the results for real measurement data as described in III.A, where the TOA difference for each transducer was computed as the median of the transducer itself and its closest neighbors to improve SNR. The model is sensitive to nonGaussian noise caused by inaccurate peak detection, especially for multiple waves arriving close to each other in time via multiple paths. This introduces higher probability of fouling along some paths (artifact). Nevertheless, we were able to locate the fouling approximately. We used $l=5.0 \mathrm{~cm}$ and $o^{2}=1.0$ for both experiments, and $s^{2}=1.0$ for artificial data and $s^{2}=5.0$ for the measured data.
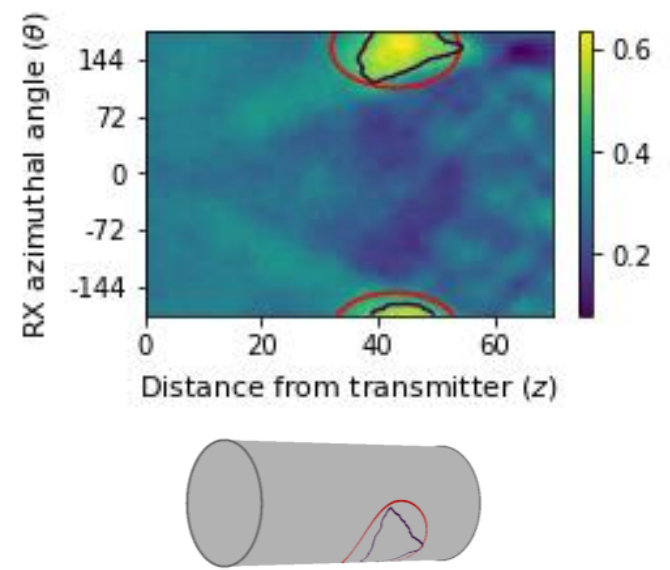

Fig. 6. Spatial distribution of the fouling probability with simulated data. High probability regions (black; $p>0.5$ for fouling thickness to exceed a threshold) accurately match the ground truth (red). Lower image shows the contours wrapped on the pipe surface to guide an eye.

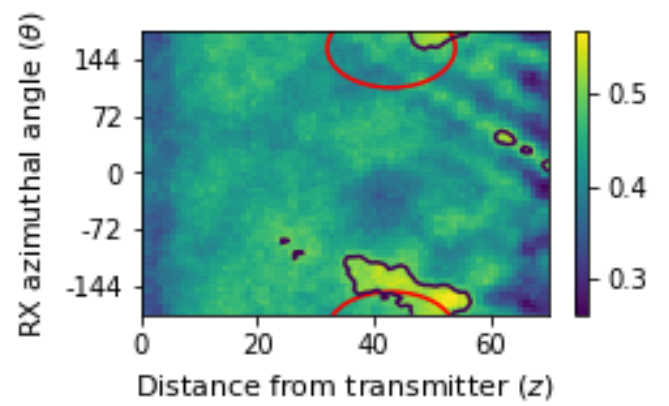

Fig. 7. Spatial distribution of the fouling probability with measurement data. High probability regions (black; $p>0.5$ ) approximately localize the ground truth (red), with some false positives.

\section{DISCUSSION AND CONCLUSIONS}

We validated the AI module on simulated data and demonstrated it in a real localization task. We approximately localized the fouling, but the method is sensitive to nonGaussian noise in peak detection. The accuracy may be improved by replacing the separate detection step by one integrated with the model, by using phase velocity changes inducted by the fouling in addition to group velocity changes, and by relaxing the normality assumption of the noise distribution and the fouling assumptions for the GP model. The results from the second measurement showed feasibility to do in situ fouling rate monitoring during cleaning.

To conclude, we showed an application of fouling detection based on helicoidal guided waves powered by machine learning.

\section{ACKNOWLEDGMENT}

The authors thank Mr. J. Suorsa (Department of Physics, University of Helsinki) for numerical simulations in this study.

\section{REFERENCES}

[1] P. B. Nagy, F. Simonetti, and G. Instanes, "Corrosion and erosion monitoring in plates and pipes using constant group velocity Lamb wave inspection.," Ultrasonics, vol. 54, no. 7, pp. 1832-1841, 2014.

[2] M. J. S. Lowe, D. N. Alleyne, and P. Cawley, "Defect detection in pipes using guided waves," Ultrasonics, vol. 36, no. 1-5, pp. 147-154, Feb. 1998.

[3] T. R. Hay and J. L. Rose, "Fouling detection in the food industry using ultrasonic guided waves," Food Control, vol. 14, no. 7, pp. 481-488, Oct. 2003.

[4] A. Arcos Jiménez, C. Q. Gómez Muñoz, and F. P. García Márquez, "Dirt and mud detection and diagnosis on a wind turbine blade employing guided waves and supervised learning classifiers," Reliability Engineering \& System Safety, vol. 184, pp. 2-12, Apr. 2019.

[5] E. Dehghan-Niri and S. Salamone, "A multi-helical ultrasonic imaging approach for the structural health monitoring of cylindrical structures," Structural Health Monitoring, vol. 14, no. 1, pp. 73-85, Jan. 2015.

[6] A. Golato, F. Ahmad, S. Santhanam, and M. G. Amin, "Multi-Helical Path Exploitation in Sparsity-Based Guided-Wave Imaging of Defects in Pipes," J Nondestruct Eval, vol. 37, no. 2, p. 27, Mar. 2018.

[7] P. Huthwaite and M. Seher, "Robust helical path separation for thickness mapping of pipes by guided wave tomography," IEEE Transactions on Ultrasonics, Ferroelectrics, and Frequency Control, vol. 62, no. 5, pp. 927-938, May 2015.

[8] C. L. Willey, F. Simonetti, P. B. Nagy, and G. Instanes, "Guided wave tomography of pipes with high-order helical modes," NDT \& E International, vol. 65, pp. 8-21, Jul. 2014.

[9] K. L. J. Fong, "A study of curvature effects on guided elastic waves," 2005.

[10] T. Kundu, Ultrasonic and Electromagnetic NDE for Structure and Material Characterization: Engineering and Biomedical Applications. CRC Press, 2016.

[11] "Dispersion Curves for a Fluid-Filled Elastic Pipe," COMSOL Multiphysics®. [Online]. Available: https://www.comsol.com/model/dispersion-curves-for-a-fluid-filledelastic-pipe-53431. [Accessed: 28-Aug-2019].

[12] C. E. Rasmussen and C. K. I. Williams, Gaussian processes for machine learning. Cambridge, Mass: MIT Press, 2006.

[13] Z. Purisha, C. Jidling, N. Wahlström, S. Särkkä, and T. B. Schön, "Probabilistic approach to limited-data computed tomography reconstruction," Inverse Problems, Jul. 2019.

[14] C. Jidling, N. Wahlström, A. Wills, and T. B. Schön, "Linearly constrained Gaussian processes," in Advances in Neural Information Processing Systems 30, I. Guyon, U. V. Luxburg, S. Bengio, H. Wallach, R. Fergus, S. Vishwanathan, and R. Garnett, Eds. Curran Associates, Inc., 2017, pp. 1215-1224.

[15] F. H. Wittmann, "Estimation of the modulus of elasticity of calcium hydroxide," Cement and Concrete Research, vol. 16, no. 6, pp. 971-972, Nov. 1986.

[16] K. Persson, "Materials Data on $\mathrm{Ca}(\mathrm{HO}) 2$ (SG:164) by Materials Project." LBNL Materials Project; Lawrence Berkeley National Laboratory (LBNL), Berkeley, CA (United States), 2014. 